\title{
Non-perturbative approach to correlations in two-dimensional vortex liquids
}

\author{
Joonhyun Yeo and M. A. Moore \\ Department of Physics, University of Manchester, Manchester, M13 9PL, United Kingdom.
} (June 25, 2021)

\begin{abstract}
We calculate the renormalized quartic vertex function of the Ginzburg-Landau model for a superconducting film in a magnetic field by summing an infinite subset of diagrams, the so-called parquet graphs. Using this non-perturbative solution, we obtain the structure factor of the two-dimensional vortex liquid. We find growing crystalline order in the system as the temperature is lowered. Our results suggest that the length scale characterizing the crystalline order diverges only in the zerotemperature limit, which indicates the absence of a finite-temperature phase transition to the vortex lattice phase.
\end{abstract}

PACS: 74.60.Ge,74.20.De

The effect of thermal fluctuations in type II superconductors in the presence of a magnetic field has been a focus of theoretical and experimental interest since the discovery of high $T_{c}$ superconductors. Mean field theory predicts the existence of the mixed phase where the magnetic field penetrates the sample in the form of flux lines which form a triangular array known as the Abrikosov vortex lattice [1]. Thermal fluctuations are believed to be responsible for the melting of the Abrikosov vortex lattice into a vortex liquid state. The melting of the two-dimensional vortex lattice has been studied in association with the dislocation unbinding mechanism leading to a continuous transition [2]. However, the question whether the two-dimensional vortex liquid undergoes a phase transition at all into the low-temperature ordered state is still controversial. The high temperature perturbation expansions of the Ginzburg-Landau (GL) model within the lowest Landau level (LLL) approximation have been analyzed for evidence of the transition to the ordered phase at low temperatures [3]. Some numerical simulations [1] of the same model revealed a weak firstorder transition between vortex liquid-solid phases. On the other hand, the Monte-Carlo (MC) simulation performed in Ref. [5] on a spherical geometry showed no sign of a finite temperature transition.

In this Letter, we present a non-perturbative analysis of the GL model for a superconducting film in a magnetic field within the LLL approximation. We make a resummation of an infinite number of diagrams summing all the parquet graphs [6]. We find a clear indication of growing crystalline order in the two-dimensional vortex liquid as the temperature is lowered. However, within our approximation, we find no evidence for a finite-temperature phase transition. In fact, our results are consistent with a zero-temperature scaling argument [5] according to which the length scale characterizing the spatial order in the vortex liquid diverges only in the zero temperature limit.

A different kind of non-perturbative scheme for the same model has been employed by Tešanović and co- workers [7] to calculate various thermodynamic quantities. This method, however, contains an adjustable parameter which is the generalized Abrikosov ratio, $\beta_{A}$. In the present nonperturbative analysis, where no adjustable parameters are used, all the physical quantities including $\beta_{A}$ are calculated as functions of temperature in a straightforward manner.

The starting point of our analysis is the GinzburgLandau free energy for a superconducting film in a perpendicular magnetic field $\mathbf{B}=\nabla \times \mathbf{A}$ given by

$F[\Psi]=\int d^{2} \mathbf{r}\left(\alpha|\Psi|^{2}+\frac{\beta}{2}|\Psi|^{4}+\frac{1}{2 m}\left|\left(-i \hbar \nabla-e^{*} \mathbf{A}\right) \Psi\right|^{2}\right)$,

where $\alpha, \beta$ and $m$ are phenomenological parameters. For a thin film superconductor, fluctuations in the vector potential A can be ignored. We restrict the order parameter $\Psi$ to the space spanned by the LLL wavefunctions. This approximation is valid near the upper critical field $H_{c_{2}}$. In the symmetric gauge, where $\mathbf{A}=\frac{B}{2}(-y, x)$, the LLL is fully described by an arbitrary holomorphic function of the variable $z=x+i y$ multiplied by an exponential factor: $\Psi(x, y)=\exp \left(-\frac{\mu^{2}}{4} z^{*} z\right) \phi(z)$, where $\mu \equiv \sqrt{e^{*} B / \hbar}$ is the inverse magnetic length. In the LLL approximation, the GL free energy becomes

$F[\phi]=\int d z^{*} d z\left\{\alpha_{H} e^{-\frac{\mu^{2}}{2}|z|^{2}}|\phi(z)|^{2}+\frac{\beta}{2} e^{-\mu^{2}|z|^{2}}|\phi(z)|^{4}\right\}$,

where $\int d z^{*} d z \equiv \int d^{2} \mathbf{r}$ and $\alpha_{H} \equiv \alpha+\hbar e^{*} B / 2 m=0$ is the point where the mean field instability occurs. The temperature will be represented by a dimensionless parameter, $\alpha_{T} \equiv \alpha_{H} \sqrt{2 \pi / \beta \mu^{2}}$. In the high temperature perturbation theory, one uses $x \equiv \mu^{2} \beta / 4 \pi \alpha_{R}^{2}$ as a dimensionless expansion parameter, where $\alpha_{R}$ denotes the renormalized $\alpha_{H}$ (i.e. the denominator of the $\left\langle\Psi^{*} \Psi\right\rangle$ propagator). The zero-temperature limit corresponds to $\alpha_{T} \rightarrow-\infty$ or $x \rightarrow \infty$. The parameter $\alpha_{R}$ is usually estimated via the Hartree approximation, where $\alpha_{R}=\alpha_{H}(1-4 x)^{-1}$ [3]. 
Using our non-perturbative method, we will be able to obtain a much improved estimate for $x$ and $\alpha_{R}$.

In the renormalization group analysis of the generalization of (11) in $6-\epsilon$ dimensions, Brézin et al. [8] indicated that the GL hamiltonian in (11) is not closed under renormalization. The renormalization drives the quartic vertex of (11) to the most general gauge invariant form, $\int \prod_{i=1,2} d z_{i}^{*} d z_{i} e^{-\frac{\mu^{2}}{2}\left(\left|z_{1}\right|^{2}+\left|z_{2}\right|^{2}\right)}\left|\phi\left(z_{1}\right)\right|^{2} g\left(\mid z_{1}-\right.$ $\left.z_{2} \mid\right)\left|\phi\left(z_{2}\right)\right|^{2}$, for an arbitrary function $g$. In this Letter, we use the Fourier transform representation [9] of the quartic vertex instead of the one used in Ref. [8]: $\widetilde{g}(k)=\int d^{2} \mathbf{r} g(r) \exp (i \mathbf{k} \cdot \mathbf{r})$. It is convenient for later use to introduce the scaled function $f(k) \equiv$ $(2 / \beta) \exp \left(-k^{2} / 2 \mu^{2}\right) \widetilde{g}(k)$. Note that the bare interaction corresponds to $f_{B}(k)=\exp \left(-k^{2} / 2 \mu^{2}\right)$.

In the present work, we obtain the renormalized quartic vertex function, $f_{R}(k)$ of the GL hamiltonian by summing all the parquet graphs [6]. We then calculate the structure factor of the two-dimensional vortex liquid from this non-perturbative solution. The structure factor denoted by $\Delta(q)$ is proportional to the Fourier transform of the spatial correlation function of the superfluid density, $\tilde{\chi}(q)=\int d^{2} \mathbf{R} e^{i \mathbf{q} \cdot \mathbf{R}} \chi(\mathbf{r}, \mathbf{r}+\mathbf{R})$, where $\chi\left(\mathbf{r}, \mathbf{r}^{\prime}\right) \equiv\left\langle|\Psi(\mathbf{r})|^{2}\left|\Psi\left(\mathbf{r}^{\prime}\right)\right|^{2}\right\rangle-\left\langle|\Psi(\mathbf{r})|^{2}\right\rangle\left\langle\left|\Psi\left(\mathbf{r}^{\prime}\right)\right|^{2}\right\rangle$ :

$$
\tilde{\chi}(q)=\frac{\mu^{2}}{2 \pi \alpha_{R}^{2}} e^{-q^{2} /\left(2 \mu^{2}\right)} \Delta(q) .
$$

The structure factor of the two-dimensional vortex liquid has been studied using the numerical simulations [4, the density-functional theory of vortex liquid freezing 10 and the high-temperature perturbation theory [11]. In terms of $f_{R}(k), \Delta(q)$ is given by

$$
\Delta(Q)=1-2 x\left\{f_{R}(Q)+\int_{0}^{\infty} d K K J_{0}(Q K) f_{R}(K)\right\},
$$

where $J_{0}$ is the Bessel function and $\mathbf{Q}=\mathbf{q} / \mu$ and $\mathbf{K}=\mathbf{k} / \mu$ are dimensionless wave vectors. Eq. (3) is the central quantity of our investigation. In (3), the first term comes from contracting two pairs of external lines in the disconnected four-point Feynman diagram. The remaining terms represent the two independent ways of contracting the connected four-point diagrams.

Using $f_{R}(K)$, one can also express $\alpha_{R}$ in terms of $\alpha_{H}$. The relation between the renormalized parameter $\alpha_{R}$ (or $x$ ) and $\alpha_{H}$ (or $\alpha_{T}$ ) gives the specific heat of the system. In the present analysis, this relation comes from the Dyson-Schwinger equation associated with the hamiltonian (11), which expresses the renormalized propagator in terms of the bare propagator and the renormalized fourpoint function. It follows that

$$
\alpha_{T}=\frac{1}{\sqrt{2 x}}\left[1-4 x\left\{1-2 x \int_{0}^{\infty} d K K f_{R}(K) e^{-K^{2} / 2}\right\}\right] .
$$

The Hartree approximation therefore corresponds to neglecting the term that depends on $f_{R}(K)$. The generalized Abrikosov ratio is defined by $\beta_{A}(x) \equiv$ $\overline{\left\langle|\Psi(\mathbf{r})|^{4}\right\rangle} /\left[\overline{\left\langle|\Psi(\mathbf{r})|^{2}\right\rangle}\right]^{2}$, where the bar denotes the spatial average. In terms of $f_{R}(K)$, we have

$$
\beta_{A}(x)=2\left[1-2 x \int_{0}^{\infty} d K K f_{R}(K) e^{-K^{2} / 2}\right] .
$$

It is interesting to see if $\beta_{A}(x)$ really converges to 1.16 as $x \rightarrow \infty$ or $\alpha_{T} \rightarrow-\infty$.

In order to calculate $f_{R}(K)$, we consider the Feynman diagrams contributing to the connected four-point function, $W\left(z_{1}^{*}, z_{2}^{*}, z_{3}, z_{4}\right) \equiv\left\langle\phi^{*}\left(z_{1}^{*}\right) \phi^{*}\left(z_{2}^{*}\right) \phi\left(z_{3}\right) \phi\left(z_{4}\right)\right\rangle_{c}$. We then make the parquet graph analysis on $W$ and obtain the corresponding relations for $f_{R}(K)$ to which $W$ is directly related within the LLL approximation [8,9]. The four-point vertex function depends on three variables characterizing the three independent channels. Since $f_{R}(K)$ is defined through the faithful representation of diagrams, we express all the diagrams involved in the parquet decomposition for $W$ in the faithful representation.

Since an arbitrary diagram contributing $W$ is either reducible or irreducible for a given channel $i=1,2$ or 3 , one can write in terms of $f_{R}(K)$ that $f_{R}(K)=I_{i}(K)+$ $\Gamma_{i}(K)$, where $I_{i}$ and $\Gamma_{i}$ are the irreducible and reducible parts for channel $i$, respectively. The irreducible part $I_{i}(K)$ consists of the bare vertex function $f_{B}(K)$, the totally irreducible part $R(K)$, which is irreducible in all channels, and those which are reducible in the other two channels. Thus, we have

$$
I_{i}(K)=f_{B}(K)+R(K)+\sum_{j \neq i} \Gamma_{j}(K) .
$$

Therefore,

$$
f_{R}(K)=f_{B}(K)+R(K)+\sum_{i=1}^{3} \Gamma_{i}(K)
$$

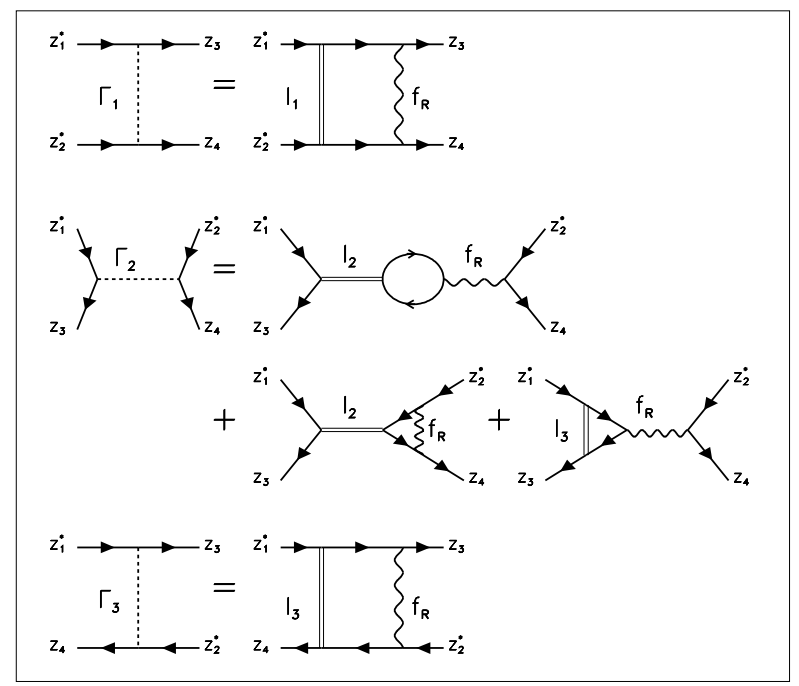

FIG. 1. Diagrammatic representation of the Bethe-Salpeter equation for the reducible parts $\Gamma_{i}(K)$. 
The reducible part $\Gamma_{i}(K)$ can be represented in terms of the Bethe-Salpeter equation as described graphically in Fig. 1. Essentially, they express the reducible part in terms of a series of ladder graphs composed of appropriate irreducible parts. The detailed derivation of these relations will be presented in a separate publication [12. By explicitly evaluating each diagram in Fig. 1, we obtain

$$
\begin{aligned}
& \Gamma_{1}(K)=-2 x \int \frac{d^{2} \mathbf{P}}{2 \pi} I_{1}(|\mathbf{K}-\mathbf{P}|) \\
& \quad \times f_{R}(P) \cos (\mathbf{K} \times \mathbf{P}) \\
& \begin{aligned}
& \Gamma_{2}(K)=-2 x\left\{I_{2}(K) f_{R}(K)\right. \\
&+I_{2}(K) \int \frac{d^{2} \mathbf{P}}{2 \pi} f_{R}(P) \cos (\mathbf{K} \times \mathbf{P}) \\
&\left.+f_{R}(K) \int \frac{d^{2} \mathbf{P}}{2 \pi} I_{3}(P) \cos (\mathbf{K} \times \mathbf{P})\right\} \\
& \Gamma_{3}(K)=-2 x \int \frac{d^{2} \mathbf{P}}{2 \pi} I_{3}(|\mathbf{K}-\mathbf{P}|) f_{R}(P),
\end{aligned}
\end{aligned}
$$

where $\mathbf{K} \times \mathbf{P} \equiv K_{1} P_{2}-K_{2} P_{1}$. It is important to note that the coupled integral equations, (6) with (5) and (7) are exact relations for $f_{R}(K)$. In the present work, we neglect the contribution from the nonparquet diagrams represented by $R(K)$ in (5), for which a systematic analysis is very difficult. We note that the lowest order diagram contributing to $R(K)$ is of $O\left(\beta^{4}\right)$. The subset of parquet graphs contain an enormous number of diagrams and we assume that the essential properties of the vortex liquid can be represented by this subset. We solve numerically eqs. (5), (5) and (7) for $f_{R}(K)$ with the condition $R(K)=0$ and evaluate $\Delta(K)$ using (3). For given set of functions, $\left\{I_{i}(K)\right\}$, Eq. (6) is a linear equation for $f_{R}(K)$ (a Fredholm equation of the second kind) which can be solved by a numerical inversion of a matrix [13]. The solution is then found by iteration with the improved irreducible parts calculated from (5) using the solution to the linear equation just obtained.

We obtain $f_{R}(K)$ and $\Delta(K)$ for various values of $x$. Before presenting our results for $\Delta(K)$, we first discuss how the perturbative expansion parameter $x$ depends on dimensionless temperature $\alpha_{T}$. From our numerical data, we find a significant improvement over the Hartree approximation. We also find that at low temperatures the $\sqrt{x}$ vs. $\alpha_{T}$ curve approaches a straight line, the slope of which is related to the Abrikosov ratio in the zero temperature limit. We can in fact plot directly the Abrikosov ratio as a function of temperature using (4). (See Fig. 2.) The value of $\beta_{A}$ decreases with decreasing temperature from its high temperature limit $\beta_{A}=2$ representing the uncorrelated vortex liquid. As one goes into the low temperature region, $\beta_{A}$ decreases very slowly. Although it is hard to determine the exact asymptotic value at $\alpha_{T}=-\infty$ from the present data, these values are getting close to 1.16 , the value for a triangular lattice.

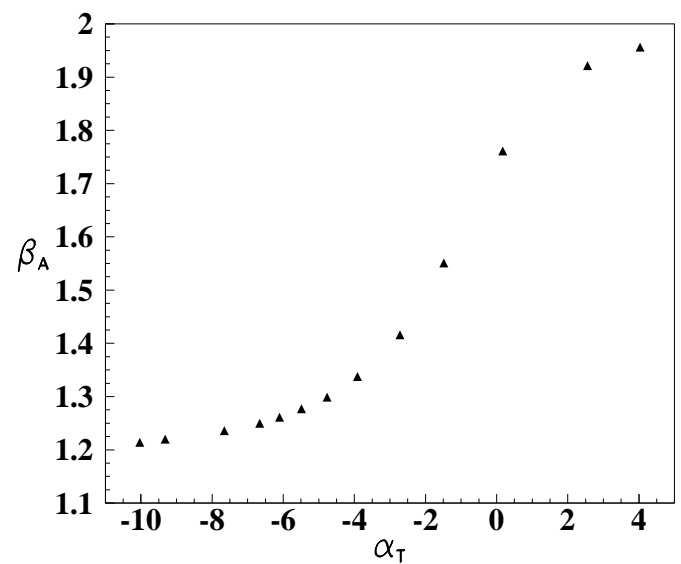

FIG. 2. The generalized Abrikosov ratio as a function of temperature.

Fig. 3 shows the structure factor $\Delta(K)$ for various temperatures. We find that, as $\alpha_{T} \lesssim-2$, the structure factor starts to develop a well-defined peak near the first reciprocal lattice vector (RLV) of a triangular lattice, $G_{1} / \mu \simeq 2.69$. As the temperature is lowered further, more structures are revealed as the peaks around the next RLVs begin to appear. Since the second and third RLVs of the triangular lattice are closely spaced, our solution could not resolve these peaks up to the minimum temperature we have considered. However, one can clearly see that the height of the first peak grows while the width narrows down as the temperature is lowered, which indicates the growing crystalline order in the two-dimensional vortex liquid system.

In Fig. 3, our nonperturbative results for $\Delta(K)$ are compared with the recent MC simulation data [14]. Up to low temperatures, they are in good agreement with the MC results. But we find that, as one goes further into the low temperature regime, the peak height in the $\mathrm{MC}$ results grows faster than in the present calculation. It is to be expected that the diagrams omitted from the parquet approximation will produce quantitative errors at low temperatures, whereas at high temperatures their effects are negligible. In Ref. 11], the structure factor of the two-dimensional vortex liquid has been evaluated up to 12th order of perturbation theory. The extrapolation to low temperatures via Padé approximants shows a peak starting to appear near the first RLV of the triangular lattice, but the method becomes unreliable at low temperatures and at small wave vectors.

In the completely ordered state where vortices form a lattice, the structure factor would exhibit delta-function like divergences at the reciprocal lattice vectors (RLV). In fact, if we use the mean field solution for $\Psi(\mathbf{r})$ [1] in the definition of the structure factor, we get $\Delta(\mathbf{k})=$ $(2 \pi)^{2} \sum_{\mathbf{G} \neq 0} \delta^{(2)}(\mathbf{k}-\mathbf{G})$, where the sum is over the RLVs of the triangular lattice except $\mathbf{G}=0$. The $\mathbf{G}=0$ term corresponds to the disconnected piece substracted in the 


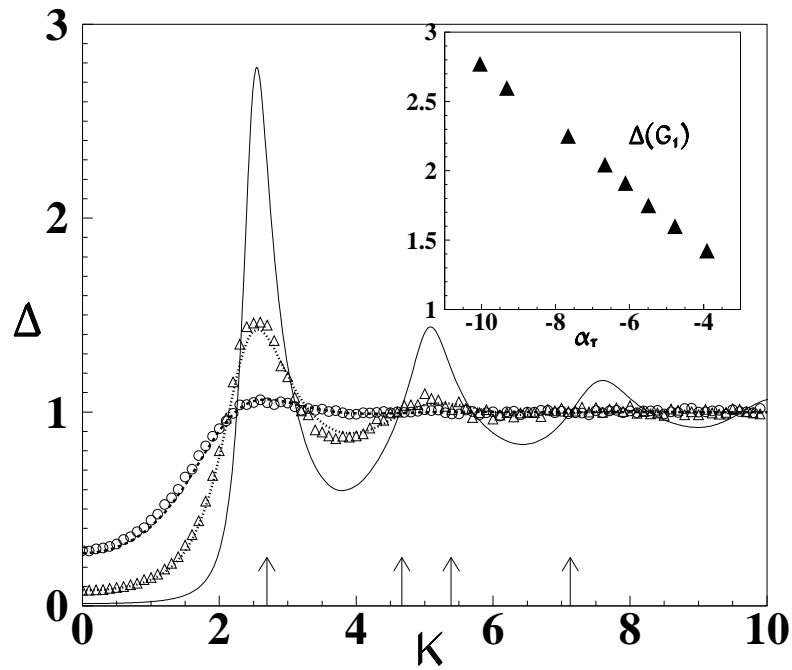

FIG. 3. The structure factor $\Delta(K)$ calculated from the present non-perturbative method for various temperatures; $\alpha_{T}=-1.5$ (the broken line), -3.9 (the dotted line) and -10 (the solid line). The arrows indicate the positions of the RLVs of the triangular lattice. The open circles and the open triangles are those obtained in the MC simulation for $\alpha_{T}=-1.5$ and -4.0 , respectively. The inset shows the height of the first peak obtained from the value of $\Delta$ at the first RLV, $G_{1}$ as a function of temperature.

definition of $\chi$. Assuming that $\Delta(K)$ obtained from our calculation approach this low temperature limit, we investigate the temperature dependence of the height of the growing peaks in the structure factor. From our numerical solution, we find that in the low temperature regime the height of the first peak grows as $\left|\alpha_{T}\right|$. (See the inset in Fig. 3.) This result can be understood in terms of the zero-temperature scaling argument [5] mentioned before. This argument is based on the observation that the destruction of off-diagonal long range order below four dimensions 15] by thermal fluctuations might be accompanied by the destruction of the positional order characterized by a length scale $\xi$. In this argument, $\xi$ diverges in the zero-temperature limit as $\left|\alpha_{T}\right|$. If we can describe $\Delta(k)$ near a peak $G$ by the function $\xi h(\xi(k-G))$, then the integral of $\Delta(k)$ over $\mathbf{k}$ around $G$ is finite as $\xi \rightarrow \infty$ [12], which is required by the asymptotic form of $\Delta(k)$. Therefore, the peak height at $k=G$ grows as $\xi \sim\left|\alpha_{T}\right|$ as $\alpha_{T} \rightarrow-\infty$.

We note that our non-perturbative approximation captures this asymptotic behavior suggested by the zero temperature scaling. From (3), we deduce that in the zero temperature limit, $f_{R}(\mathbf{k}) \sim(2 x)^{-1}\left\{1-\pi \mu^{2} \sum_{\mathbf{G}} \delta^{(2)}(\mathbf{k}-\right.$ G)\}. If we put this expression into eqs. (5), (6) and (7) and focus on the region $\mathbf{k} \sim \mathbf{G} \neq 0$, we find $(4 \pi / x) \delta^{(2)}(\mathbf{k}-\mathbf{G})=$ a smooth function of $\mathbf{k}$ around $\mathbf{G}$ independent of $x$. Upon integrating over the region with the peak width $\xi^{-1}$, we find $\xi^{2} \sim x \sim \alpha_{T}^{2}$ as $\alpha_{T} \rightarrow-\infty$. This argument indicates that our data for $\Delta(K)$ already exhibit the asymptotic behavior suggested by the zero- temperature scaling and therefore within our approximation the phase transition to the vortex lattice state at finite temperature is highly unlikely.

To summarize, we have found an analytic approach to the vortex liquid regime which is sophisticated enough to predict, $a b$ initio, the growth of crystalline order as the temperature is lowered; which agrees qualitatively with $\mathrm{MC}$ estimates of the structure factor at moderate to high temperatures; while in the low-temperature regime it is consistent with zero-temperature scaling argument, and the absence of a finite temperature phase transition.

We thank T.Newman for useful discussions and M.Dodgson for providing his MC simulation data.

[1] A.A.Abrikosov, Zh. Eskp. Theo. Fiz. 32, 1442 (1957) [Sov. Phys. JETP 5, 1174 (1957)].

[2] S.Doniach and B.A.Huberman, Phys. Rev. Lett. 42, 1169 (1979), D.S.Fisher, Phys. Rev. B 22, 1190 (1980).

[3] G.J.Ruggeri and D.J.Thouless, J. Phys. F 6, 2063 (1976), R.Ikeda, T.Ohmi and T.Tsuneto, J. Phys. Soc. Jpn. 59, 1397 (1990), E.Brézin, A.Fujita and S.Hikami, Phys. Rev. Lett. 65, 1949 (1990), S.Hikami, A.Fujita and A.I.Larkin, Phys. Rev. B 44, 10400 (1991).

[4] Z.Tešanović and L.Xing, Phys. Rev. Lett. 67, 2729 (1991). Y.Kato and N.Nagaosa, Phys. Rev. B 47, 2932 (1993), J.Hu and A.H.MacDonald, Phys. Rev. Lett. 71, 432 (1993), R.Š́sik and D.Stroud, Phys. Rev. B 49, 16074 (1994).

[5] J.A.O'Neill and M.A.Moore, Phys. Rev. B 48, 374 (1993).

[6] See for example A.I.Larkin and D.E.Khmel'nitskii, Zh. Eksp. Theo. Fiz. 56, 2087 (1969) [Sov. Phys. JETP 29, 1123 (1969)], B.Roulet, J.Gavoret and P.Nozières, Phys. Rev. 178, 1027 (1969), T.Tsuneto and E.Abrahams, Phys. Rev. Lett. 30, 217 (1973).

[7] Z.Tešanović et al., Phys. Rev. Lett. 69, 3563 (1992), Z.Tešanović and A.V.Andreev, Phys. Rev. B 49, 4064 (1994).

[8] E.Brézin, D.R.Nelson and A.Thiaville, Phys. Rev. B 31, 7124 (1985).

[9] M.A.Moore and T.J.Newman, preprint.

[10] S.Sengupta et al., Phys. Rev. Lett. 67, 3444 (1991), I.F.Herbut and Z.Tešanović, Phys. Rev. Lett. 73, 484 (1994).

[11] J.Hu, A.H.MacDonald and B.D.McKay, Phys. Rev. B 49, 15263 (1994).

[12] J.Yeo and M.A.Moore, in preparation.

[13] W.H.Press et al., Numerical Recipes, p.782, Cambride University Press, Cambridge (1992).

[14] M.J.W.Dodgson and M.A.Moore, unpublished.

[15] M.A.Moore, Phys. Rev. B 39, 136 (1989), ibid. 45, 7336 (1992). 